\title{
A SALTING-OUT ASSISTED LIQUID-LIQUID EXTRACTION FOR THE DETERMINATION OF TEBUCONAZOLE, CARBOFURAN AND IMIDACLOPRID IN WHITE AND ROSÉ WINES
}

\author{
Fernando J. G. Daglia, Josiele A. M. Conrado ${ }^{a}$, Luiz André J. Silva ${ }^{a}$ and Alex D. Batista ${ }^{\mathrm{a}, *(\mathbb{0})}$ \\ anstituto de Química, Universidade Federal de Uberlândia, 38400-902 Uberlândia - MG, Brasil
}

Recebido em 18/03/2020; aceito em 30/04/2020; publicado na web em 18/06/2020

\begin{abstract}
Food safety is a constant and necessary concern due to the worldwide increase of the food demand. The monitoring of quality parameters demands the development of accurate, easy and fast procedures. The aim of this work was to develop a cheap, miniaturized and trustworthy salting-out assisted liquid-liquid extraction (SALLE) procedure for the determination of pesticides in wines. Sample and acetonitrile were mixed and the addition of $\mathrm{NaCl}$ and $\mathrm{CH}_{3} \mathrm{COONa}$ were used for separation of aqueous and organic phases. After centrifugation, the organic phase was directly injected into a liquid chromatograph. The developed SALLE procedure consumed at least 25-fold less reagent compared to conventional QuEChERS procedure. Additionally, the d-SPE step of the QuEChERS method was suppressed, without efficiency losses. The developed procedure was compared to the conventional methodology and no significant difference was observed. It was successfully applied to the determination of three pesticides in wines by liquid chromatography. Low consumption of sample and solvent, low residues generation, simple instrumentation are notable advantages. The suppression of the d-SPE step simplified the process and the risk of analyte losses.
\end{abstract}

Keywords: pesticides; liquid chromatography; sample preparation.

\section{INTRODUCTION}

Wine is an alcoholic beverage consumed worldwide, especially in European countries, such as Portugal, Italy and France, produced by the fermentation of different kind of grapes. ${ }^{1}$ Grapes production and wine quality are highly influenced by climate, which delimitates the wine production areas and increases the occurrences of plagues. Thus, the use of pesticides is almost mandatory to achieve high production and high-quality grapes. Consequently, pesticides residues have been found in wines in the range of micrograms per liter. ${ }^{2,3}$ Although pesticides are found in wines at low concentration levels, they can cause chronic effects on human health. ${ }^{4}$ Despite the increasing concern about food safety, the European Union has no specific legislation for pesticides levels in wines. ${ }^{5}$ Grapes are very susceptible to several plagues as they are produced at limited areas which increase the use of pesticides. Part of these pesticides is transferred to the wine during its production. ${ }^{6}$

A variety of pesticides have been applied to grapes for plagues control. ${ }^{7}$ Their application depends on the grape type and the region of the production. Tebuconazole is a systemic fungicide with a broad spectrum of action worldwide used in the control of pathogenic fungi in nuts, cereals, vegetables and fruits, especially in grapes. ${ }^{8}$ Imidacloprid is synthetic insecticide employed for control of insects in a variety of fruits, including grapes. Although it presents low toxicity, it can cause fertility problems in males when subjected to chronic exposure, even at low concentrations. ${ }^{9}$ In some countries with poor monitoring pesticides programs, forbidden pesticides are still employed which makes necessary the development of analytical methods for these chemicals. Carbofuran is a forbidden pesticide in U.S.A and Europe due its high toxicity and persistence on the environment. It was widely used as insecticide, nematicide and acaricide with a wide spectrum of activity against several agriculture pests. $^{10}$

Several analytical techniques have been used for the residual determination of pesticides in wines. Due to the complexity of the

*e-mail: alexbatista@ufu.br wine samples, the proposed procedures for pesticides determination usually employ separation techniques, mainly liquid and gas chromatography with different detectors. ${ }^{2}$ The complexity of the wine samples and the low pesticides concentration levels found on these samples make mandatory the use of sample preparation steps for the removal of potential interferents and preconcentration of the analytes. Several techniques have been employed for this purpose, such as a solid-phase microextraction, ${ }^{11}$ stir bar sorptive extraction, ${ }^{12}$ matrix solid-phase dispersion ${ }^{13}$ and single drop microextraction. ${ }^{14}$

In order to overcome some limitations of existing multiresidues methods, a new procedure for the extraction of pesticides in agricultural food samples was presented, and it was named as QuEChERS (quick, easy, cheap, effective, rugged and safe). ${ }^{15}$ The unnecessary use of specific instrumentations, its excellent performance and compatibility with most separation techniques popularized the QuEChERS method. ${ }^{16}$ It is based on three steps: (i) extraction with acetonitrile; (ii) partition promoted by the addition of salts and (iii) dispersive solid phase extraction. Despite the satisfactory performance of the QuEChERS procedure, it consumes a relatively high amount of organic solvents and salts and involves many manipulation steps compared to microextraction techniques.

The aim of these work was to develop a cleaner alternative sample preparation to QuEChERS protocol based on salting-out assisted liquid-liquid extraction for the extraction of tebuconazole, carbofuran and imidacloprid from white and rosé wines.

\section{EXPERIMENTAL}

\section{Chemicals and solutions}

Ultrapure water $(18 \mathrm{M} \Omega \mathrm{cm})$ was employed to prepare solutions and mobile phases. Acetonitrile ( $\geq 99.9 \%$ ) obtained from Merk was used in QuEChERS procedure and as a mobile phase component. Tebuconazole ( $\geq 98.0 \%)$, carbofuran $(\geq 98.0 \%)$ and imidacloprid $(\geq 98.0 \%)$ were purchased from Sigma Aldrich and stock solutions were prepared by dilution in methanol (HPLC grade, $\geq 99.9 \%$, from Sigma Aldrich) and working solutions by dilution in the mobile 
phase. Sodium chloride, magnesium sulphate and sodium acetate were obtained from Synth (Diadema, Brazil). Different wine samples (white and rosé wines) were obtained from local markets.

\section{Salting-out assisted liquid-liquid extraction procedure}

The SALLE was performed in a $1.5-\mathrm{mL}$ Eppendorf ${ }^{\circledast}$ tube by the adding of $0.5 \mathrm{~mL}$ of sample (rosé or white wine), $0.5 \mathrm{~mL}$ of acetonitrile followed by manual shaken during $1 \mathrm{~min} .50 \mathrm{mg}$ of sodium chloride and $150 \mathrm{mg}$ of sodium acetate were added into the flask and the mixture was shaken for during $1 \mathrm{~min}$. For the separation of organic and aqueous phases, the flask was centrifugated at $895 \mathrm{~g}$-force by 5 minutes. $100 \mu \mathrm{L}$ of the organic upper phase was collected by a microsyringe and injected into an HPLC-UV system for separation and detection.

All the optimization experiments were performed employing wines from producers that do not adopt the use of pesticides during the grape production to guarantee the absence of unknow concentrations of the target analytes during the optimization step. All the optimization experiments employed samples spiked with $500 \mu \mathrm{g} \mathrm{L}^{-1}$ of each pesticide.

\section{Instrumentation}

A centrifuge from Enterprise, model C 1008-P, was used for phase separation during LLME procedure. Chromatographic analyses were performed on a Shimadzu LC-6AD equipped with an SPDM20A diode array detector, a C18 CLC-ODS column (Phenomenex, $150 \times 4.6 \mathrm{~mm}$ d.i., $5 \mu \mathrm{m}) \mathrm{kept}$ at $35^{\circ} \mathrm{C}$. An SIL-20AC Autosampler was employed for sample injection and a LC-20AT pump for solvents delivery. Gradient elution was performed employing a mobile phase composed by acetonitrile (A) and water (B) as follow: start, $80 \% \mathrm{~B}$; $10 \mathrm{~min}, 20 \% \mathrm{~B} ; 15$ - $20 \mathrm{~min}, 80 \% \mathrm{~B}$ with a flow rate of $1.0 \mathrm{~mL} \mathrm{~min}{ }^{-1}$. Analysis time was 20 min including the chromatographic separation and column conditioning for the next analysis. Sample volume injected into the column was $20 \mu \mathrm{L}$. Detection was performed at $296 \mathrm{~nm}$ for imidacloprid and at $221 \mathrm{~nm}$ for tebuconazole and carbofuran. All the extracts were filtered in a $0.22 \mu \mathrm{m}$ PTFE filter to avoid the clogging of the chromatographic column by some solid residue from the samples. Data were evaluated at LabSolutions Software provided by Shimadzu.

\section{RESULTS AND DISCUSSIONS}

\section{Salting-out assisted liquid-liquid extraction procedure optimization}

The parameters optimization of the SALLE aimed to achieve a condition with high analytes extraction efficiency with minimum extraction of potential interference species. The salt added to the acetonitrile and wine mixture changes the physical-chemical properties of the system resulting in two phases system and partitioning the analytes preferably to the organic phase (saltingout effect). The efficiency of three different salts was evaluated as presented in Figure 1a. The binary mixture of $\mathrm{NaCl}$ and $\mathrm{CH}_{3} \mathrm{COONa}$ achieved the best performance. The first salt $(\mathrm{NaCl})$ contributes for the decreasing of the extraction of polar interferents from the matrix and improves solubility of the analytes in the organic phase, improving their extraction. ${ }^{17}$ The condition (B) without $\mathrm{NaCl}$ presented lower analytes extraction. The original QuEChERS procedure employs high amounts of salts and a high fraction of the added salts remains insoluble. ${ }^{15}$ Condition A and B presented a small amount of insoluble salts, while condition $\mathrm{C}$ (best composition) achieved complete solubilization of the salts, which indicates that the use of saturated mixtures is not mandatory to attain high analytes extraction efficiency. The proposed procedure employed 25-fold less salts without the need for saturated mixtures, which results in a cheaper and environmentally friendly procedure, as it produces less residues.

The influence of the proportion of $\mathrm{NaCl}$ and $\mathrm{CH}_{3} \mathrm{COONa}$ was evaluated (Figure 1b) and no significant differences were observed for Carbofuran extraction in the evaluated conditions. Imidacloprid presented the best extraction efficiency at the condition with a higher proportion of $\mathrm{CH}_{3} \mathrm{COONa}$ and tebuconazole at the higher proportion of $\mathrm{NaCl}$. The acetate hydrolysis increases the $\mathrm{pH}$ of the aqueous phase changing the distribution of charged and uncharged species of the analytes, which could improve or compromise the extraction into the organic phase. Thereby, the proportion of $\mathrm{NaCl}$ and $\mathrm{CH}_{3} \mathrm{COONa}$ was set at 1:3.

The original QuEChERS method employs a dispersive solid phase extraction (d-SPE) step after separation of organic and aqueous phases. This step involves the use a sorbent material, usually functionalized with a primary secondary amine (PSA) to remove polar interferences (including fatty acids, other organic acids, sugars and pigments, such as anthocyanidins). ${ }^{16}$ The efficiency of the proposed SALLE procedure was evaluated without the d-SPE step and similar responses were achieved, indicating that this step can be avoided (Figure 2). Thereby, the SALLE is faster and cheaper than the conventional QuEChERS procedure. The high performance of the proposed strategy without d-SPE step can be explained by the fact that white and rosé wines are relatively less complex matrixes compared to other food and environmental samples.

The performance of the optimized SALLE procedure was also compared to the conventional QuEChERS methodology by a statistical t-test $\left(\mathrm{t}_{\text {calculated }}=1.72, \mathrm{t}_{\text {table }}=2.13, \mathrm{n}=5\right)$ and no significant differences were observed as presented in Figure 2. The same efficiency was achieved by the proposed procedure employing
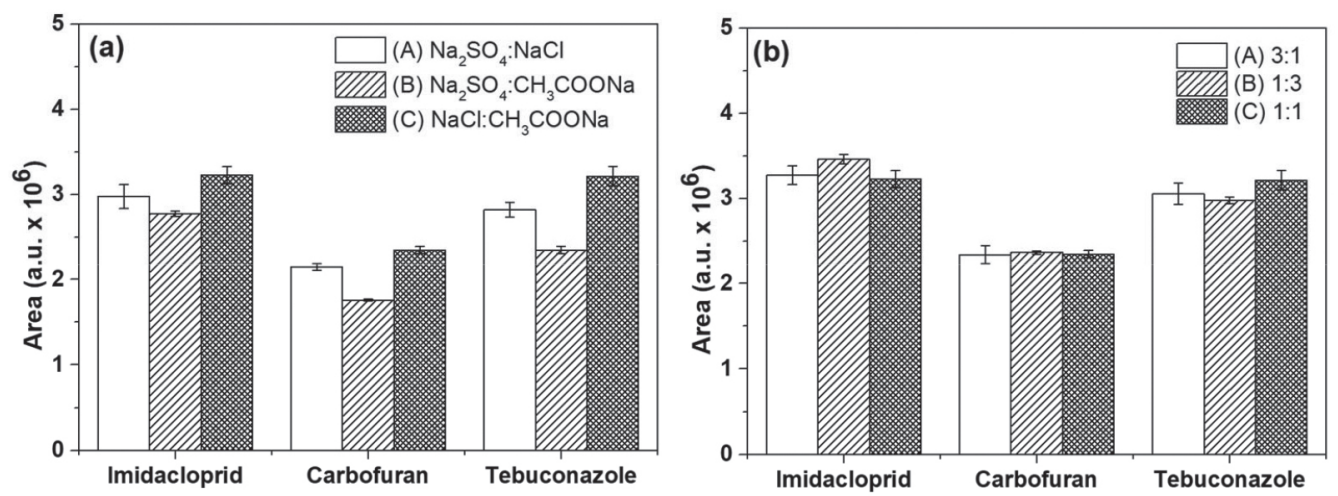

Figure 1. (a) influence of the different salts on the pesticides extraction from white wine by SALLE; (b) influence of the proportion of $\mathrm{NaCl}_{\text {and }} \mathrm{CH} \mathrm{H}_{3} \mathrm{COONa}$ on the on the extraction of pesticides from white wine by SALLE. Evaluation performed adding $500 \mu \mathrm{g} L^{-1}$ of each pesticide 


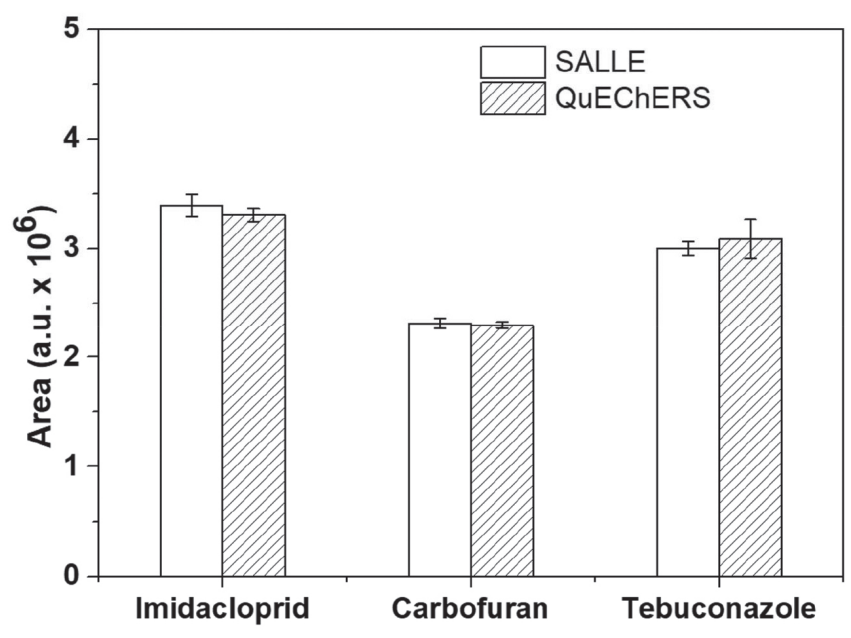

Figure 2. Comparison of the performance of conventional QuEChERS employing PSA sorbent for $d$-SPE step and the developed SALLE

25-fold less salt amount, 20-fold less acetonitrile and sample volumes, additionally to the absence of the d-SPE step. These features make the proposed strategy cheaper, faster and environmentally friendly. Chromatograms obtained by SALLE and QuEChERS are presented at Figure 3.

\section{Analytical features}

Calibrations were performed employing nine solutions with different concentrations of the pesticides and linear responses were observed from the 0.5 up to $1000.0 \mu \mathrm{g} \mathrm{L}^{-1}$ with satisfactory linear coefficients as presented at Table 1. Limits of detection and quantification were experimentally estimated by decreasing concentration until signal noise ratio above three and nine, respectively..$^{18}$ The repeatability of the SALLE was evaluated by the analysis of nine replicates of wine sampled added with $100 \mu \mathrm{g} \mathrm{L}^{-1}$ of each pesticide and it was estimated at $1.4 \%, 2.4 \%$ and $2.2 \%$ for imidacloprid, carbofuran and imidacloprid, respectively. Recovery studies at three different concentration levels were performed for accuracy evaluation as presented at Table 2. Best recoveries, in most result, were achieved for higher concentration additions. The lowest evaluated concentration provided lower recoveries values. However, most of the values are accepted according to the European Commission that defines that procedures for pesticides determination on food samples from animal or vegetal origin can present recoveries on the range of 60 to $120 \%$ with relative standards deviation up to $30 \% .{ }^{19}$ This highlights the potential of the presented procedure for the simultaneous analysis of these three pesticides. The procedure was also evaluated for the analysis of red wine samples. However, recoveries were unsatisfactory due to the more complex matrix composition.

The proposed procedure presents advantages compared to previous SALLE published procedures as shown at Table 3. It consumes smaller amount of organic solvent per analysis with one of the smallest RSD, which demonstrate a good precision of the procedure.

\section{Application to real samples}

Thirty samples (fifteen rosé wines and fifteen white wines) were analysed by the SALLE and all the pesticides were presented below the limit of detection. These samples were spiked with the pesticides and analysed again as presented at Table 4 . The results highlight the applicability of the proposed procedure to the analysis of the analytes in rosé and white wines. Despite the higher matrix complexity of the rosé wines compared to white wines, good analytical features were obtained for these samples.



Figure 3. Chromatograms from wine samples with $500 \mu \mathrm{g} L^{-1}$ of each pesticide obtained by SALLE and QuEChERS

Table 1. Analytical features of the SALLE procedure for the chromatographic determination of pesticides in wine

\begin{tabular}{lcccccccc}
\hline Pesticide & $\mathrm{a}$ & $\mathrm{b}$ & $\mathrm{r}^{2}$ & $\mathrm{RT}(\mathrm{min})$ & $\mathrm{R}_{\mathrm{s}}$ & $\mathrm{CV}(\%)$ & $\left.\mathrm{LOD}_{(\mu \mathrm{g} \mathrm{L}}{ }^{-1}\right)$ & $\left.\mathrm{LOQ}_{(\mu \mathrm{g} \mathrm{L}}{ }^{-1}\right)$ \\
\hline Imidacloprid & 2.1331 & 3468.458 & 0.999 & 6.5 & 2.14 & 1.4 & 0.096 & 0.29 \\
Carbofuran & 8.2784 & 1150.502 & 0.999 & 10.0 & 7.92 & 2.4 & 0.16 & 0.50 \\
Tebuconazole & 18.9908 & 1411.247 & 0.999 & 12.9 & 13.52 & 2.2 & 0.13 & 0.41 \\
\hline
\end{tabular}

r: correlation coefficient; a: linear coefficient; b: angular coefficient; $\mathrm{CV}$ : coefficient of variation ( $\mathrm{n}=9$ ); RT: retention time; $\mathrm{R}_{\mathrm{s}}$ : chromatographic resolution related to the closest peak. 
Table 2. Recoveries from white and rosé wine samples

\begin{tabular}{|c|c|c|c|c|}
\hline \multirow{2}{*}{ Pesticide } & \multicolumn{2}{|c|}{ White wine } & \multicolumn{2}{|c|}{ Rosé wine } \\
\hline & Added $\left(\mu \mathrm{g} \mathrm{L}{ }^{-1}\right)$ & Recovery (\%) & Added $\left(\mu \mathrm{g} \mathrm{L}^{-1}\right)$ & Recovery (\%) \\
\hline \multirow{3}{*}{ Imidacloprid } & 500 & 106 & 500 & 106 \\
\hline & 100 & 99 & 100 & 105 \\
\hline & 20 & 91 & 20 & 93 \\
\hline \multirow{3}{*}{ Carbofuran } & 500 & 94 & 500 & 99 \\
\hline & 100 & 92 & 100 & 102 \\
\hline & 20 & 89 & 20 & 93 \\
\hline \multirow{3}{*}{ Tebuconazole } & 500 & 103 & 500 & 108 \\
\hline & 100 & 97 & 100 & 100 \\
\hline & 20 & 89 & 20 & 94 \\
\hline
\end{tabular}

Table 3. Comparison of the proposed SALLE procedure to similar works previously presented

\begin{tabular}{|c|c|c|c|c|c|}
\hline Analyte / Sample & Organic solvent / volume & Salt & $\operatorname{RSD}(\%)$ & Recoveries $(\%)$ & Ref. \\
\hline Biogenic amines / Fish, meat & Acetonitrile/ $2.0 \mathrm{~mL}$ & $\mathrm{NaCl}$ & $1.3-15$ & $80-115$ & 20 \\
\hline Cyanotoxins / water & Acetonitrile/ $2.0 \mathrm{~mL}$ & $\left(\mathrm{NH}_{4}\right)_{2} \mathrm{SO}_{4}$ & $3.9-14.6$ & $61.5-99.5$ & 21 \\
\hline Insecticid / Honey & Acetonitrile/ $1.0 \mathrm{~mL}$ & $\mathrm{CH}_{3} \mathrm{COONa}$ & $5.3-3.4$ & $99.2-104.3$ & 22 \\
\hline Hydroxymethylfurfural / Honey & Acetonitrile / $3 \mathrm{~mL}$ & $\mathrm{Na}_{2} \mathrm{CO}_{3}$ & $1.6-2.9$ & $94.1-95.0$ & 23 \\
\hline Tylosin A, desmycosin / Honey & Acetonitile / $5 \mathrm{~mL}$ & $\mathrm{CH}_{3} \mathrm{COONa}$ & $<7$ & $90.2-111.2$ & 24 \\
\hline Vitamin D3 / Milk & Acetonitile / $3 \mathrm{~mL}$ & $\left(\mathrm{NH}_{4}\right)_{2} \mathrm{SO}_{4}$ & $1.6-11.4$ & $94.4-113.5$ & 25 \\
\hline Pesticides / Wine & Acetonitrile / $0.5 \mathrm{~mL}$ & $\mathrm{NaCl} / \mathrm{CH}_{3} \mathrm{COONa}$ & $1.4-2.4$ & $80-108$ & This work \\
\hline
\end{tabular}

Table 4. Analysis of rosé and white wines spiked with $100 \mu \mathrm{g} \mathrm{L} \mathrm{L}^{-1}$ of each pesticide by the SALLE procedure

\begin{tabular}{|c|c|c|c|c|}
\hline Sample & & Imidacloprid & Carbofuran & Tebuconazole \\
\hline \multirow{15}{*}{ Rosé wine } & 1 & $105.5 \pm 2.5$ & $101.3 \pm 0.9$ & $102.8 \pm 2.4$ \\
\hline & 2 & $101.3 \pm 1.9$ & $105.9 \pm 1.1$ & $105.7 \pm 2.9$ \\
\hline & 3 & $106.9 \pm 2.1$ & $102.8 \pm 2.0$ & $101.9 \pm 1.9$ \\
\hline & 4 & $108.7 \pm 2.4$ & $109.5 \pm 1.2$ & $109.1 \pm 3.1$ \\
\hline & 5 & $103.1 \pm 1.8$ & $104.1 \pm 1.4$ & $108.6 \pm 2.6$ \\
\hline & 6 & $109.8 \pm 1.7$ & $110.3 \pm 2.3$ & $100.2 \pm 2.4$ \\
\hline & 7 & $100.5 \pm 2.0$ & $108.5 \pm 1.9$ & $107.1 \pm 2.9$ \\
\hline & 8 & $104.9 \pm 1.9$ & $105.9 \pm 0.9$ & $99.8 \pm 3.2$ \\
\hline & 9 & $107.4 \pm 2.4$ & $103.8 \pm 1.8$ & $107.5 \pm 1.7$ \\
\hline & 10 & $99.8 \pm 1.8$ & $107.5 \pm 1.3$ & $102.7 \pm 2.3$ \\
\hline & 11 & $101.8 \pm 2.8$ & $102.8 \pm 1.7$ & $101.4 \pm 2.7$ \\
\hline & 12 & $106.5 \pm 1.7$ & $105.1 \pm 2.4$ & $108.8 \pm 3.2$ \\
\hline & 13 & $102.8 \pm 0.9$ & $104.7 \pm 2.2$ & $100.2 \pm 1.6$ \\
\hline & 14 & $100.3 \pm 1.9$ & $108.4 \pm 1.5$ & $103.5 \pm 2.0$ \\
\hline & 15 & $98.6 \pm 2.8$ & $107.9 \pm 2.0$ & $100.8 \pm 1.1$ \\
\hline \multirow{15}{*}{ White wine } & 16 & $109.5 \pm 2.6$ & $93.5 \pm 1.2$ & $99.1 \pm 1.2$ \\
\hline & 17 & $104.3 \pm 3.0$ & $97.9 \pm 1.9$ & $98.7 \pm 2.0$ \\
\hline & 18 & $108.3 \pm 2.1$ & $93.8 \pm 2.0$ & $101.3 \pm 1.8$ \\
\hline & 19 & $107.8 \pm 2.2$ & $91.7 \pm 2.4$ & $97.3 \pm 2.5$ \\
\hline & 20 & $101.6 \pm 2.7$ & $92.8 \pm 1.7$ & $103.7 \pm 3.0$ \\
\hline & 21 & $109.7 \pm 1.9$ & $99.3 \pm 1.3$ & $99.5 \pm 2.1$ \\
\hline & 22 & $110.1 \pm 3.0$ & $95.7 \pm 2.5$ & $104.7 \pm 1.8$ \\
\hline & 23 & $107.8 \pm 2.0$ & $100.2 \pm 2.3$ & $102.8 \pm 2.7$ \\
\hline & 24 & $109.4 \pm 2.7$ & $94.7 \pm 1.4$ & $100.3 \pm 3.2$ \\
\hline & 25 & $106.3 \pm 1.9$ & $98.8 \pm 1.8$ & $98.7 \pm 2.0$ \\
\hline & 26 & $108.7 \pm 2.0$ & $90.1 \pm 2.0$ & $103.8 \pm 2.1$ \\
\hline & 27 & $110.3 \pm 2.5$ & $99.6 \pm 2.3$ & $106 \pm 1.7$ \\
\hline & 28 & $105.7 \pm 3.1$ & $101.3 \pm 1.4$ & $97.8 \pm 3.3$ \\
\hline & 29 & $103.9 \pm 1.7$ & $95.4 \pm 2.1$ & $99.4 \pm 2.6$ \\
\hline & 30 & $107.6 \pm 2.6$ & $93.1 \pm 2.3$ & $98.9 \pm 1.5$ \\
\hline
\end{tabular}

\section{CONCLUSIONS}

A SALLE was presented for the extraction of pesticides from white and rosé wines. The proposed procedure has remarkable advantages compared to the conventional QuEChERS procedure such as significant reduction on the consumption of sample and reagents and the absence of the d-SPE step, which makes the procedure cheaper, faster and environmentally friendly compared to conventional QuEChERS methodology. These features fulfil the requirements originally proposed by the methodology that should be quick, easy, cheap, effective, robust and safe. The proposed procedure can be applied to routine analysis of carbofuran, tecobunazole and imidacloprid in white and rosé wines.

\section{ACKNOWLEDGMENTS}

This work was supported by the Brazilian governmental agencies: Conselho Nacional de Desenvolvimento Científico e Tecnológico (CNPq, Proc. 425114/2018-0), Coordenação de Aperfeiçoamento de Pessoal de Nível Superior (CAPES) and state governmental agency Fundação de Amparo à Pesquisa do Estado de Minas Gerais (FAPEMIG, Proc. APQ-02272-18).

\section{REFERENCES}

1. World Health Organisation; Global status report on alcohol and health, 2014. Available at https://apps.who.int/iris/bitstream/hand le/10665/112736/9789240692763_eng.pdf, accessed at June 2020.

2. Jin, B.; Xie, L.; Guo, Y.; Pang, G.; Food Res. Int. 2012, 46, 399.

3. Grimalt, S.; Dehouck, P.; J. Chromatogr. A 2016, 1433, 1.

4. Kim, K.-H.; Kabir, E.; Jahan, S. A.; Sci. Total Environ. 2017, 575, 525.

5. Wang, X.; Telepchak, M. J.; LC-GC Eur. 2013, 26, 66.

6. Pazzirota, T.; Martin, L.; Mezcua, M.; Ferrer, C.; Fernandez-Alba, A. R.; Food Addit. Contam., Part A 2013, 30, 1752.

7. Angioni, A.; Dedola, F.; Environ. Monit. Assess. 2013, 185, 4281.

8. You, X.; Li, Y.; Wang, X.; Xu, J.; Zheng, X.; Sui, C.; Biomed. Chromatogr. 2017, 31, e3917. 
9. Bal, R.; Türk, G.; Tuzcu, M.; Yilmaz, O.; Kuloglu, T.; Gundogdu, R.; Gür, S.; Agca, A.; Ulas, M.; Çambay, Z.; Tuzcu, Z.; Gencoglu, H.; Guvenc, M.; Ozsahin, A. D.; Kocaman, N.; Aslan, A.; Etem, E.; $J$. Environ. Sci. Health, Part B 2012, 47, 434.

10. dos Anjos, J. P.; de Andrade, J. B.; Microchem. J. 2015, 120, 69.

11. Ravelo-Pérez, L. M.; Hernández-Borges, J.; Borges-Miquel, T. M.; Rodríguez-Delgado, M. Á.; Food Chem. 2008, 111, 764.

12. Maggi, L.; Zalacain, A.; Mazzoleni, V.; Alonso, G. L.; Salinas, M. R.; Talanta 2008, 75, 753 .

13. Albero, B.; Sánchez-Brunete, C.; Tadeo, J. L.; J. Agric. Food Chem. 2003, 51, 6915.

14. Augusto, F.; Carasek, E.; Silva, R. G. C.; Rivellino, S. R.; Batista, A. D.; Martendal, E.; J. Chromatogr. A 2010, 1217, 2533.

15. Anastassiades, M.; Lehotay, S. J.; Štajnbaher, D.; Schenck, F. J.; $J$. AOAC Int. 2003, 86, 412.

16. González-Curbelo, M. Á.; Socas-Rodríguez, B.; Herrera-Herrera, A. V.; González-Sálamo, J.; Hernández-Borges, J.; Rodríguez-Delgado, M. Á.; TrAC Trends Anal. Chem. 2015, 71, 169.

17. Correia-Sá, L.; Fernandes, V. C.; Carvalho, M.; Calhau, C.; Domingues, V. F.; Delerue-Matos, C.; J. Sep. Sci. 2012, 35, 1521.
18. Wenzl, T.; Haedrich, J.; Schaechtele, A.; Robouch, P.; Stroka, J.; Burdaspal, P.; Kapp, T.; Amlund, H.; Jensen, U.; Patriarca, M.; Gambin, M. D.; Nuotio, K.; Christou, E.; Mol, H.; Debongnie, P.; Cuhra, P.; Guidance document on the estimation of $L O D$ and $L O Q$ for measurements in the field of contaminants in feed and food, 2016, http:// dx.doi.org/10.2787/8931.

19. European Commission, 2010, available at https://ec.europa.eu/food/sites/ food/files/plant/docs/pesticides_ppp_app-proc_guide_res_post-reg-contmonitor.pdf, accessed at June 2020.

20. Francisco, K. C. A.; Brandão, P. F.; Ramos, R. M.; Gonçalves, L. M.; Cardoso, A. A.; Rodrigues, J. A.; Int. J. Food Sci. Technol. 2020.

21. Hemmati, M.; Tejada-Casado, C.; Lara, F. J.; García-Campaña, A. M.; Rajabi, M.; del Olmo-Iruela, M.; J. Chromatogr. A 2019.

22. Giroud, B.; Bruckner, S.; Straub, L.; Neumann, P.; Williams, G. R.; Vulliet, E.; Microchem. J. 2019.

23. Chen, W.; Wu, S.; Zhang, J.; Yu, F.; Miao, X.; Tu, X.; Anal. Methods 2019.

24. Thompson, T. S.; van den Heever, J. P.; Komarnicki, J. A. F.; Anal. Bioanal. Chem. 2019.

25. Sazali, N. H.; Alshishani, A.; Saad, B.; Chew, K. Y.; Chong, M. M.; Miskam, M.; R. Soc. Open Sci. 2019. 